\title{
Evaluation of the retinal nerve fibre layer and ganglion cell complex thickness in pituitary macroadenomas without optic chiasmal compression
}

G Cennamo ${ }^{1}$, RS Auriemma², D Cardone 1 , LFS Grasso ${ }^{3}$, N Velotti' ${ }^{1}$ C Simeoli3 , C Di Somma ${ }^{3}$, R Pivonello ${ }^{3}, \mathrm{~A} \mathrm{Colao}^{3}$ and $\mathrm{G}$ de Crecchio

\begin{abstract}
Purpose The aim of this prospective study was to measure the thickness of the circumpapillary retinal nerve fibre layer (cpRNFL) and the ganglion cell complex (GCC) using spectral domain optical coherence tomography (SD-OCT) in a cohort of consecutive de novo patients with pituitary macroadenomas without chiasmal compression. Patients and methods Twenty-two consecutive patients with pituitary macroadenoma without chiasmal compression (16 men, 6 women, aged $45.2 \pm 14.6$ years, 43 eyes) entered the study between September 2011 and June 2013. Among them, $31.8 \%$ harboured a growth hormone-secreting pituitary adenoma, 27.3\% a prolactin-secreting pituitary adenoma, $27.3 \%$ a corticotrophin-secreting pituitary adenoma, and $13.6 \%$ a non-secreting pituitary tumour. Eighteen subjects (nine females, nine males, mean age $36.47 \pm 6.37$ years; 33 eyes) without pituitary adenoma, with normal ophthalmic examination, served as controls. In both patients and controls, cpRNFL and GCC thicknesses were measured by SD-OCT.

Results Patients were significantly older $(P=0.02)$ than controls. Best corrected visual acuity, intraocular pressure, colour fundus photography, and automatic perimetry test were within the normal range in patients and controls. Conversely, cpRNFL $(P=0.009)$ and GCC $(P<0.0001)$ were significantly thinner in patients than in controls. The average GCC $(r=0.306, P=0.046)$ significantly correlated with the presence of arterial hypertension. OCT parameters did not differ significantly between patients with a tumour volume
\end{abstract}

above the median and those with a tumour volume below the median.

Conclusion Pituitary macroadenomas, even in the absence of chiasmal compression, may induce GCC and retinal nerve fibre layer thinning. SD-OCT may have a role in the early diagnosis and management of patients with pituitary tumours.

Eye (2015) 29, 797-802; doi:10.1038/eye.2015.35; published online 27 March 2015

\section{Introduction}

Pituitary tumours are common intracranial neoplasms, accounting for up to $10-15 \%$ of intracranial tumours at surgery and $6-23 \%$ at autopsy. ${ }^{1,2}$ They comprise $20 \%$ of all primary brain and central nervous system tumours and represent the second most common type identified by histology in young adults (20-34 years) according to the Central Brain Tumor Registry of the USA. ${ }^{3}$ A population-based study conducted in the province of Liège, Belgium, reported a 3-5 times higher prevalence of clinically relevant pituitary tumours (1:1064) than previously estimated. ${ }^{4}$ Pituitary adenomas are generally associated with hormone overproduction and/or compression signs and symptoms due to the impingement of adjacent structures, including optic chiasm. ${ }^{5-11}$

Symptoms are related to their size and position: if the adenoma is larger than $10 \mathrm{~mm}$ in diameter ('macroadenoma') it can extend beyond the limits of the sella and can cause neuroophthalmologic disorders by compressing the adjoining structures. ${ }^{1,12}$ Abnormalities in the retinal nerve fibre layer (RNFL) in long-standing lesions are also characteristic of pituitary
${ }^{1}$ Dipartimento di Neuroscienze, Scienze Riproduttive ed Odontostomatologiche, University of Naples'Federico II', Naples, Italy

${ }^{2}$ Coleman-los Medicina Futura Medical Center, Naples, Italy

${ }^{3}$ Dipartimento di Medicina Clinica e Chirurgia, Sezione di Endocrinologia, University of Naples'Federico II', Naples, Italy

Correspondence:

G Cennamo, Dipartimento di Neuroscienze, Scienze Riproduttive ed Odontostomatologiche, University of Naples 'Federico II', via S. Pansini 5, 80131 Naples, Italy Tel: +39 (0)817463731; Fax: +39 (0)817462384 E-mail: xgilda@hotmail.com

Received: 10 June 2014 Accepted in revised form: 3 February 2015 Published online: 27 March 2015 
tumours. ${ }^{6}$ Measurement of the circumpapillary retinal nerve fibre layer (cpRNFL) and the ganglion cell complex (GCC) by spectral domain optical coherence tomography (SD-OCT) may predict visual outcome. The GCC includes the RNFL, retinal ganglion cell (RGC) layers, and inner plexiform layer. ${ }^{13}$ Previous studies ${ }^{14-18}$ of patients with large pituitary tumours that induce optic chiasm compression identified a diffuse loss of RNFLs, even in the absence of visual field defects, and hypothesised that pituitary tumours per se, even in the absence of a compressive effect on the chiasm, may cause reversible RGC dysfunction. Whether pituitary tumours not associated to optic chiasm compression may also induce damage in RNFL is unknown. The aim of this prospective study was to evaluate cpRNFL and GCC thicknesses measured by SD-OCT in a cohort of consecutive de novo patients with pituitary macroadenomas without chiasmal compression.

\section{Materials and methods}

Twenty-two consecutive patients ( 16 men, 6 women, aged $45.2 \pm 14.6$ years, 43 eyes) with a newly established diagnosis of pituitary macroadenoma, admitted to the Eye Clinic and Neuroendocrine Disease Unit of the University of Naples 'Federico II' between September 2011 and June 2013, entered this prospective study. The main inclusion criteria were: age $>18$ years; evidence at pituitary magnetic resonance imaging (MRI) of a pituitary macroadenoma without chiasm impingement; written informed consent to study participation. Exclusion criteria were: any previous treatment for pituitary adenoma; presence of congenital eye disorders; myopia $>6$ dioptres; a history of ocular surgery; significant lens opacities or any macular disease; a previous diagnosis of glaucoma; and any optic disc anomaly. Eighteen patients (nine women, nine men, aged $44.68 \pm 13.73$ years) without pituitary adenoma and with a normal ophthalmic examination served as the control group. On the basis of power calculation and sample size analysis, a total of 16 eyes were required for a statistical power of $80 \%$ at a significance set at $5 \%$.

The study protocol was approved by the institutional review board of the University of Naples Federico II and adhered to the tenets of the Declaration of Helsinki.

Written informed consent was obtained from each subject after explanation of the study.

At study entry, a medical history and duration of symptoms were recorded for each patient. All patients harboured a pituitary macroadenoma at the study entry. Mean tumour volume at baseline was $3900.8 \pm 7404.5 \mathrm{ml}$ (range 40.8-32487 ml). Tumour volume was above the median $(1256.6 \mathrm{ml})$ in $11 / 22(50 \%)$ and below the median in $11 / 22(50 \%)$. Patients were classified on the basis of hormonal hypersecretion associated to pituitary tumours: growth hormone $(\mathrm{GH})$-secreting pituitary tumours (no $=7,31.8 \%)$, prolactin (PRL)-secreting pituitary tumours (no=6, 27.3\%); corticotrophin (ACTH)-secreting pituitary tumours (no $=6,27.3 \%)$; and non-secreting pituitary tumours (NFA, no $=3,13.6 \%$ ). Data about previous diagnoses, current treatment of arterial hypertension, and abnormalities in glucose metabolism (impaired glucose tolerance or overt diabetes mellitus) were also collected.

All patients underwent a complete ophthalmic examination including best-corrected visual acuity (BCVA) according to the standard Early Treatment of Diabetic Retinopathy Study (LogMAR), intraocular pressure (IOP), colour fundus photography, and the automatic perimetry test (Humphrey Field Analyzer using the 30-2 SITA-Standard algorithm of the Humphrey perimeter; Carl Zeiss Meditec, Dublin, CA, USA). The Humphrey Field Analyzer 30-2 tests 76 points in the central 30 degrees of the visual field. The thicknesses of GCC and cpRNFL were evaluated with SD-OCT (RTVue-100 OCT, Optovue Inc., Fremont, CA, USA; software version 4.0.5.39). The optic nerve head map protocol was used to evaluate cpRNFL thickness. This protocol generates a cpRNFL thickness map based on measurements obtained along a circle $3.45 \mathrm{~mm}$ in diameter centred on the optic disc. Only high-quality images, as defined by a signal strength index above 30, were accepted. GCC thickness was measured from the internal limiting membrane to the outer boundary of the inner plexiform layer. The device software generates a significance map with normative database comparison for GCC thickness. SD-OCT evaluations were performed by one experienced ophthalmologist blinded to patient's data.

Statistical analysis was performed using the Statistical Package for Social Sciences (version 21.0, SPSS Inc., Chicago, IL, USA). The unpaired Student $t$-test was used to compare the patients' eyes with control eyes. The non parametric Mann-Whitney test was made to compare patients with tumour volume above and those with tumour volume below the median. The comparison of numerical data between the various groups of patients classified according to hormonal hypersecretion was performed by Kruskal-Wallis test. Prevalences were compared using the $\chi^{2}$-test corrected by the Fisher's exact test, if necessary. The correlation study was done by calculating Pearson's correlation coefficients. A $P$-value $<0.05$ was considered statistically significant.

\section{Results}

At study entry, 9 out of the 22 patients (40.9\%), namely 3 patients with GH-secreting, 2 with PRL-secreting and 4 with ACTH-secreting tumours, had systemic arterial 
hypertension, defined as systolic blood pressure $>130 \mathrm{~mm} \mathrm{Hg}$ and diastolic blood pressure $>90 \mathrm{~mm} \mathrm{Hg}$ according to guidelines of the American Society of Hypertension. ${ }^{19}$ Based on plasma glucose levels, impaired glucose tolerance and diabetes mellitus were diagnosed according to WHO guidelines. ${ }^{20}$ Impaired glucose tolerance was found in $6 / 22$ patients $(27.3 \%)$, including 4 patients with GH-secreting, 1 with PRL-secreting and 1 with ACTH-secreting tumours. Overt diabetes mellitus was recorded in $8 / 22$ patients $(36.4 \%)$, including 1 patient with GH-secreting, 3 with PRL-secreting and 4 with ACTH-secreting tumours. Overall, eight (36.4\%) patients had both arterial hypertension and glucose abnormalities, seven $(31.8 \%)$ had only arterial hypertension or only glucose abnormalities, and seven (31.8\%) had neither arterial hypertension nor glucose abnormalities.

BCVA, IOP, mean deviation, and pattern SD were similar in patient and control groups (Table 1). Small isolated defects were present only in three eyes (7\%) of patients with adenoma. Conversely, focal loss volume $(P=0.004)$ and global loss volume $(P<0.0001)$ were significantly higher in patients than in healthy control subjects (Table 2$)$. Average GCC $(P<0.0001)$, and average cpRNFL $(P=0.009)$ were significantly lower in patients than in controls (Table 2). GCC and RNFL thicknesses were reduced in $82 \%$ and in $69 \%$ of patients, respectively.

Table 1 Characteristics of the study population

\begin{tabular}{lccc}
\hline & $\begin{array}{c}\text { Patients } \\
\text { group }\end{array}$ & Control & P-value \\
\hline Participants, $n$ & 22 & 18 & \\
Eyes, $n$ & 43 & 36 & \\
Age, years & $45.3 \pm 14.9$ & $44.68 \pm 13.73$ & 0.7 \\
Male & 16 & 9 & \\
Female & 6 & 9 & \\
IOP, mmHg & $16.14 \pm 2.01$ & $16.67 \pm 1.59$ & 0.15 \\
Mean deviation, $\mathrm{dB}^{\mathrm{a}}$ & $0.23 \pm 0.86$ & $-1.08 \pm 0.33$ & 0.23 \\
Pattern standard deviation, & $1.77 \pm 0.43$ & $2.12 \pm 0.62$ & 0.3 \\
$\mathrm{~dB}^{\mathrm{a}}$ & & & \\
\hline
\end{tabular}

${ }^{a}$ Obtained with the 30-2 SITA-Standard algorithm of the Humphrey perimeter.
Overall, RNFL and GCC thinning was found in 24/43 eyes $(57.7 \%)$ and in none of the controls $(P<0.0001)$. Patients with GH-secreting tumours had a significantly lower average cpRNFL $(P=0.040)$ compared with patients affected by ACTH-secreting adenomas, and in the superior hemi-field the cpRNFL was significantly lower compared with patients affected by PRL- $(P=0.040)$ and ACTH-secreting tumours $(P=0.030)$ (Table 3$)$. NFA patients had superior hemi-field cpRNFL significantly lower as compared with both patients with $P R L$ $(P=0.032)$ and ACTH- $(P=0.041)$ secreting tumours (Table 3). No significant difference was found in OCT parameters between patients with a tumour volume above and those with a tumour volume below the median. A trend towards a slight but not significant difference $(P=0.06)$ was found in cPRNFL in patients with both arterial hypertension and glucose abnormalities as compared with those with neither arterial hypertension or glucose abnormalities (Table 4). Average GCC ( $r=0.306, P=0.046)$ was significantly correlated with the presence of arterial hypertension, whereas a trend towards a significant correlation $(r=0.29, P=0.057)$ was found between average cpRNFL and a diagnosis of hypertension. Noteworthy, no significant correlation was found between any OCT parameter and presence of impaired glucose tolerance or overt diabetes mellitus.

\section{Discussion}

This prospective study reports the use of SD-OCT in the largest series of patients with different histotypes of pituitary adenoma published to date, all without chiasmal compression. Of the 22 patients evaluated, all 43 eyes showed good visual acuity and a normal visual field. GCC and cpRNFL thicknesses were reduced in $82 \%$ and in $69 \%$ of patients, respectively. Overall, cpRNFL and GCC thinning were significantly more frequent in patients than in controls, suggesting that pituitary adenomas per se might exert a direct action in damaging RNFLs and might contribute to progressive visual loss.

Table 2 Ganglion cell complex thickness and cpRNFL thickness in the patient group and in the control group

\begin{tabular}{lccc}
\hline & Patient group & Control group & P-value \\
\hline GCC average $(\mu \mathrm{m})$ & $92.1 \pm 9$ & $99.8 \pm 7.2$ & $P<0.0001$ \\
GCC FLV $(\%)$ & $1.7 \pm 2.78$ & $0.4 \pm 0.6$ & $P=0.004$ \\
GCC GLV $(\%)$ & $8.1 \pm 6.9$ & $3 \pm 2.2$ & $P<0.0001$ \\
GCC superior $(\mu \mathrm{m})$ & $91.8 \pm 8.8$ & $99.6 \pm 5.5$ & $P<0.0001$ \\
GCC inferior $(\mu \mathrm{m})$ & $92.4 \pm 9.7$ & $99.1 \pm 6.3$ & $P<0.0001$ \\
RNFL superior hemisphere $(\mu \mathrm{m})$ & $102.6 \pm 15.6$ & $113.5 \pm 24.2$ & $P=0.027$ \\
RNFL inferior hemisphere $(\mu \mathrm{m})$ & $100.8 \pm 13.6$ & $115.4 \pm 27.7$ & $P<0.007$ \\
RNFL average $(\mu \mathrm{m})$ & $101.7 \pm 13.4$ & $114.2 \pm 24.5$ & $P<0.009$ \\
\hline
\end{tabular}

Abbreviations: cpRNFL, circumpapillary retinal nerve fibre layer; FLV, focal loss volume; GCC, ganglion cell complex; GLV, global loss volume. 
Table 3 Ganglion cell complex thickness and cpRNFL thickness in patients with different subtypes of pituitary tumours

\begin{tabular}{|c|c|c|c|c|c|c|c|c|c|c|}
\hline & $\begin{array}{c}G H \text {-secreting } \\
\text { (A) }\end{array}$ & $\begin{array}{c}\text { PRL-secreting } \\
\text { (B) }\end{array}$ & $\begin{array}{c}\text { ACTH-secreting } \\
\text { (C) }\end{array}$ & $N F A(D)$ & $\begin{array}{c}P \\
(A \text { vs } B)\end{array}$ & $\begin{array}{c}P \\
(A \text { vs } C)\end{array}$ & $\begin{array}{c}P \\
(A \text { vs } D)\end{array}$ & $\begin{array}{c}P \\
(B \text { vs } C)\end{array}$ & $\begin{array}{c}P \\
(B \text { vs } D)\end{array}$ & $\begin{array}{c}P \\
\text { (C vs } D\end{array}$ \\
\hline No. $(\%)$ & $7(31.8)$ & $6(27.3)$ & $6(27.3)$ & $3(13.6)$ & & & & & & \\
\hline Eyes no. & 13 & 12 & 12 & 6 & & & & & & \\
\hline GCC average & $89.7 \pm 10.2$ & $93.7 \pm 6.6$ & $93.8 \pm 6.8$ & $87.6 \pm 12.3$ & 0.57 & 0.57 & 0.83 & 1 & 0.49 & 0.49 \\
\hline GCC superior & $88.8 \pm 10.8$ & $93.7 \pm 5.8$ & $94.5 \pm 7.6$ & $89.4 \pm 10.6$ & 0.22 & 0.29 & 0.89 & 0.79 & 0.38 & 0.25 \\
\hline GCC inferior & $90.6 \pm 9.9$ & $93.7 \pm 7.6$ & $96 \pm 6.7$ & $85.8 \pm 15$ & 0.44 & 0.17 & 0.70 & 0.51 & 0.49 & 0.44 \\
\hline RNFL average & $95.4 \pm 15.4$ & $107.4 \pm 10.6$ & $107.2 \pm 12.1$ & $96 \pm 12.5$ & 0.07 & 0.04 & 0.89 & 0.93 & 0.12 & 0.07 \\
\hline RNFL superior & $96.1 \pm 18.6$ & $109.6 \pm 12$ & $108.7 \pm 15.9$ & $95.2 \pm 12.4$ & 0.04 & 0.03 & 0.89 & 1 & 0.032 & 0.041 \\
\hline RFNL inferior & $94.8 \pm 14.1$ & $105.2 \pm 13.6$ & $105.8 \pm 10.1$ & $96.8 \pm 15.3$ & 0.25 & 0.07 & 0.83 & 0.67 & 0.44 & 0.25 \\
\hline
\end{tabular}

Abbreviation: NFA, non functioning pituitary adenoma.

Table 4 Circumpapillary retinal nerve fibre layer in patients with both arterial hypertension and glucose abnormalities as compared with those with neither arterial hypertension or glucose abnormalities

\begin{tabular}{lcclccccccc}
\hline & $H T(A)$ & $D M(B)$ & $H T+D M(C)$ & $N o H T / D M(D)$ & $P$ & $p$ & $P$ & $P$ & $P$ & $P$ \\
$(A$ vs $B)$ & $(B$ vs $C)$ & $(A$ vs $C)$ & $(B$ vs $D)$ & $(A$ vs $D)$ & $(C$ vs $D)$ \\
\hline cpRNFL & $111165 \pm 14.98$ & $111.16 \pm 12.34$ & $103.8 \pm 13.58$ & $95.1 \pm 12.77$ & 0.132 & 0.95 & 0.45 & 0.125 & 0.132 & 0.06 \\
CGG & $94.11 \pm 5.38$ & $95.3 \pm 8.9$ & $93.07 \pm 6.9$ & $87.9 \pm 10.92$ & 0.687 & 0.97 & 0.49 & 0.134 & 0.179 & 0.56 \\
\hline
\end{tabular}

Abbreviations: DM, diabetes mellitus; HT, arterial hypertension.

Previous studies have evaluated cpRNFL and GCC thickness in patients with pituitary adenomas, specifically in those with chiasmal compression. Stratus OCT revealed a diffuse loss of RNFLs particularly in the nasal and temporal aspects of the optic nerve in two patients with long-standing bitemporal visual field defects. ${ }^{14}$ Using the same technique, Johansson and Lindblom noted a reduction of cpRNFL that did not correlate with the visual field defect. ${ }^{15}$ In 19 patients, Jacob et al ${ }^{16}$ demonstrated that cpRNFL thinning measured by stratus OCT decreased the patient's chances of recovery of initial visual field defect 3 months after treatment. Inferior cpRNFL was strongly prognostic of visual field outcome after treatment of anterior visual pathway compressive lesions. Ventura et $a l^{17}$ reported that pituitary tumours, even in the absence of a compressive effect at the chiasm on MRI, may cause reversible RGC dysfunction, which precedes visual field loss and RGC death as shown by cPRNFL-OCT. Moreover, visual field recovery and evidence of changes in RGC after chiasmal decompression have been shown to be significantly correlated. ${ }^{18}$

In our prospective study, we found GCC and cpRNFL thinning in $\sim 58 \%$ of patients with pituitary adenoma without chiasmal compression and with a normal visual field and good visual acuity (Figure 1). The presence of a pituitary tumour, even in the absence of compressive effect at the chiasm on MRI, may cause GCC and cpRNFL thinning, which precedes visual field loss. Moreover, in our case series, average cpRNFL and superior cpRNFL thickness differed significantly between secreting and non-secreting tumours. Interestingly, in patients with GH-secreting pituitary tumours, the average cpRNFL was significantly lower than in those with ACTH-secreting adenomas, and superior $c p R N F L$ significantly lower than in patients with PRL-and ACTH-secreting tumours. Similarly, NFA patients had a significantly lower cpRNFL superior as compared with both patients with PRL- and ACTHsecreting tumours. Moreover, in our patient cohort, GCC average was significantly and directly related to the presence of hypertension, regardless of tumour histotype. There was a slight, albeit not significant, correlation between RNFL and prevalence of hypertension. These findings are consistent with a recent report of a significantly lower ganglion cell-inner plexiform layer $(\mathrm{GC}+\mathrm{IPL})$ in patients with arterial hypertension than in controls. ${ }^{21}$

In our series, it is possible that micro-compression was missed by the MRI. Alternatively, the GCC loss might be related to interference in axoplasmic flow induced by local ischaemia at the level of optic chiasm..$^{22}$ This hemodynamic perturbation might be related to the action of vasoactive peptide endothelin-1 that is released by pituitary tumours. ${ }^{23}$ GCC and RNFL dysfunction, measured by SD-OCT, has also been demonstrated in early glaucoma. ${ }^{24}$ The difference between these two conditions is that in glaucoma, the presumed insult (elevated IOP and other factors) acts on the retina and optic nerve head, whereas in pituitary tumours, the 

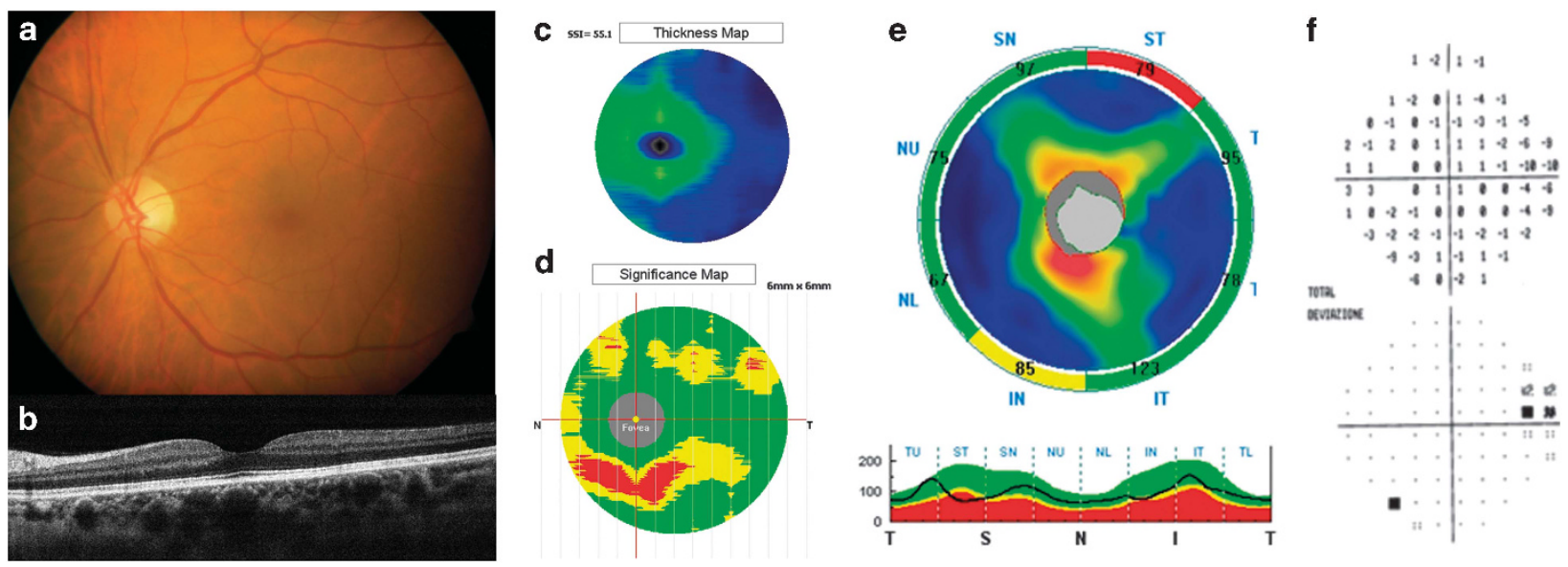

Figure 1 (a) Colour fundus image of eye with pituitary adenoma without chiasmal compression shows normal bilateral macula and optic discs. (b) A longitudinal OCT B-scan passing through the fovea shows normal macular profile. (d) Reduction of GCC thickness (c) at significance map with normative database comparison and (d) at GCC thickness map. The Significance Map is colour-coded where green represents values within the normal range ( $P$-value $5-95 \%)$, yellow indicates borderline results $(P$-value $0.5 \%)$, and red represents outside normal limits $(P$-value $<1 \%)$. (e) cpRNFL thickness is also reduced in the superotemporal sector. (f) Perimetry shows an isolated defect temporal to the fovea.

presumed insult acts at a retrobulbar location. It is possible that impairment of axoplasmic transport may contribute to both conditions. However, even though the control group was age-matched with patients, we cannot exclude that age had a role in the different GCC thicknesses between the two groups.

Noteworthy, in our patient cohort the presence of other metabolic co-morbidities, such as impaired glucose tolerance and overt diabetes mellitus, known to lead to the characteristic proliferative retinopathy that affects $\sim 30 \%$ of diabetic patients according to largest series published to date (15326 patients), ${ }^{25}$ did not further reduce GCC and cpRNFL thickness. These findings are consistent with previous data showing that in patients with diabetes mellitus, dysfunction of the inner retina is associated with a progressive increase in retinal thickening and a sponge-like appearance of the retinal layers, ${ }^{26}$ until the development of macular oedema.

In conclusion, our study suggests that GCC and RNFL thinning detected with SD-OCT may be present in patients with pituitary tumours even in the absence of chiasmal compression and may be associated with preserved visual acuity. Therefore, SD-OCT may have a role in the early diagnosis and management of patients with pituitary tumours and in the discrimination between secreting and non-secreting pituitary tumours. Additional longitudinal studies are needed to verify and to understand the findings reported herein. The continuing development of SD-OCT techniques will be useful for the early diagnosis and follow-up of pituitary adenoma.

\section{Summary}

What was known before

- cPRNFL have been evaluated in pituitary macroadenomas with optic chiasmal compression.

What this study adds

- Reduction of cpRNFL and ganglion cell complex may be present in patients with macroadenomas without optic chiasmal compression and may be associated with preserved visual acuity.

\section{Conflict of interest}

The authors declare no conflict of interest.

\section{References}

1 Melmed S, Kleinberg D. Anterior Pituitary. In: Melmed S, Polonsky KS, Reed Larsen P (eds). Williams Textbook of Endocrinology, 11th ed. Elsevier Health Sciences: Philadelphia, 2007.

2 Kovacs K, Horvath E. Pathology of pituitary tumors. Endocrinol Metab Clin North Am 1987; 16: 529-551.

3 Central Brain Tumor Registry of the United States 2007-2008. Central Brain Tumor Registry of the United States Statistical Report, 2008n 2006.

4 Daly AF, Rixhon M, Adam C, Dempegioti A, Tichomirowa MA, Beckers A. High prevalence of pituitary adenomas: a cross-sectional study in the province of Liège, Belgium. J Clin Endocrinol Metab 2006; 91: 4769-4775.

5 Colao A, Di Somma C, Pivonello R, Faggiano A, Lombardi G, Savastano S. Medicaltherapy for clinically non-functioningpituitaryadenomas. Endocr Relat Cancer 2008; 15(4): 905-915.

6 Cappabianca P, Alfieri A, Colao A, Ferone D, Lombardi G, de Divitiis E. Endoscopic endonasaltranssphenoidal approach: 
an additional reason in support of surgery in the management of pituitary lesions. Skull Base Surg 1999; 9(2): 109-117.

7 Colao A, Pivonello R, Auriemma RS, Briganti F, Galdiero M, Tortora $\mathrm{F}$ et al. Predictors of tumor shrinkage after primary therapy with somatostatin analogs in acromegaly: a prospective study in 99 patients. J Clin Endocrinol Metab 2006; 91(6): 2112-2218.

8 Colao A, Di Sarno A, Cappabianca P, Briganti F, Pivonello R, Somma CD et al. Gender differences in the prevalence, clinical features and response to cabergoline in hyperprolactinemia. Eur J Endocrinol 2003; 148(3): 325-331.

9 Pivonello R, Matrone C, Filippella M, Cavallo LM, Di Somma $\mathrm{C}$, Cappabianca $\mathrm{P}$ et al. Dopamine receptor expression and function in clinically nonfunctioning pituitary tumors: comparison with the effectiveness of cabergoline treatment. J Clin Endocrinol Metab 2004; 89(4): 1674-1683.

10 Colao A, Savastano S. Medical treatment of prolactinomas. Nat Rev Endocrinol 2011; 7(5): 267-278.

11 Colao A, Di Sarno A, Landi ML, Cirillo S, Sarnacchiaro F, Facciolli $G$ et al. Long-term and low-dose treatment with cabergoline induces macroprolactinoma shrinkage. J Clin Endocrinol Metab 1997; 82(11): 3574-3579.

12 Zee CS, Go JL, Klim PE, Mitchell D, Ahmadi J. Imaging of the pituitary and parasellar region. Neurosurg Clin N Am 2003; 14: 55.

13 Monteiro ML, Leal BC, Rosa AA. Optical coherence analysis of axonal loss in band atrophy of optic nerve. Br J Ophthalmol 2004; 88: 896-899.

14 Leal BC, Moura FC, Monteiro ML. Retinal nerve fiber layer loss documented by Stratus OCT in patients with pituitary adenoma: case report. Arq Bras Oftalmol 2006; 69: 251-254.

15 Johansson C, Lindblom B. The role of optical coherence tomography in the detection of pituitary adenoma. Acta Ophthalmol 2009; 87: 776-779.

16 Jacob M, Raverot G, Jouanneau E, Borson-Chazot F, Perrin G, Rabilloud $\mathrm{M}$ et al. Predicting visual outcome after treatment of pituitary adenomas with optical coherence tomography. Am J Ophthalmol 2009; 147: 64-70.

17 Ventura LM, Venzara FX, Porciatti V. Reversible dysfunction of retinal ganglion cells in non-secreting pituitary tumors. Doc Ophthalmol 2009; 118: 155-162.
18 Moon CH, Hwang SC, Ohn YH, Park TK. The time course of visual field recovery and changes of retinal ganglion cells after optic chiasmal decompression. Invest Ophthalmol Vis Sci 2011; 52: 7966-7973.

19 Chobanian AV, Bakris GL, Black HR, Cushman WC, Green LA, IzzoJr JL et al. Joint National Committee on Prevention, Detection, Evaluation, and Treatment of High Blood Pressure. National Heart, Lung, and Blood Institute; National High Blood Pressure Education Program Coordinating Committee. The seventh report of the Joint National Committee on Prevention, Detection, Evaluation, and Treatment of High Blood Pressure: the JNC 7 report. Hypertension 2003; 42: 1206-1252.

20 World Health Organization. Definition, diagnosis and classification of diabetes mellitus and its complications: report of a WHO Consultation. Part. 1. Diagnosis and classification of diabetes mellitus. World Health Organization: Geneva, 1999.

21 Francoz M, Fenolland JR, Giraud JM, El Chehab H, Sendon D, May F et al. Reproducibility of macular ganglion cell- inner plexiform layer thickness measurement with cirrus HD-OCT in normal, hypertensive and glaucomatous eyes. $\mathrm{Br} \mathrm{J}$ Ophthalmol 2014; 98: 322-328.

22 Cioffi GA. Ischemic model of optic nerve injury. Trans Am Ophthalmol Soc 2005; 103: 592-613.

23 Lange M, Pagotto U, Hopfner U, Ehrenreich H, Oeckler R, Sinowatz $\mathrm{F}$ et al. Endothelin expression in normal human anterior pituitaries and pituitary adenomas. J Clin Endocrinol Metab 1994; 79(6): 1864-1870.

24 Ventura LM, Porciatti V. Restoration of retinal ganglion cell function in early glaucoma after intraocular pressure reduction: a pilot study. Ophthalmology 2005; 112: 20-27.

25 Pelletier EM, Shim B, Ben-Joseph R, Caro JJ. Economic outcomes asso-ciated with microvascular complications of type 2 diabetes mellitus: results from a US claims data analysis. Pharmacoeconomics 2009; 27: 479-490.

26 Alkuraya H, Kangave D, Abu El-Asrar AM. The correlation between coherence tomography features and severity of retinopathy, macular thickness and visual acuity in diabetic macular edema. Int Ophthalmol 2005; 26(3): 93-99. 